\title{
Anesthetic implications for the hispanic population
}

Volume 9 Issue I - 2017

\section{Introduction}

Cultural awareness is a critical aspect of supporting the emotional, psychological, and physiological aspects of surgical patients. Knowing how to respect individuals for their beliefs and values is an excellent way to improve patient satisfaction and provide top level anesthesia. According to the United States (U.S.) Census Bureau, ${ }^{1}$ the Hispanic population is the second fastest growing U.S. minority. The Hispanic population now accounts for 17.1 percent of the total U.S. population. ${ }^{2}$ The Hispanic population, as defined by the U.S. Census Bureau, refers to a person of Cuban, Mexican, Puerto Rican, Central American, South America except for Brazil, or other Spanish culture or origin regardless of race (2012).

It is important for anesthesia providers to know any differences or important anesthetic considerations about the target population to provide optimal care. There is currently a tremendous amount of diversity within the Hispanic population. ${ }^{3}$ The basics for the Hispanic culture include aspects of personalismo, (personal/genuine relationships), respecto (respect), confianza (trust/confidence), and familiaismo (familial/group emphasis). The Hispanic population's overall health often has multiple factors that have impact, including language barriers, lack of access of preventive care, and lack of health insurance. ${ }^{4}$ An anesthetist's awareness of Hispanic belief system including religion, familism, language, natural remedies, and physical differences among ethnic groups can have a positive impact on the quality of anesthesia delivered.

\section{Culture beliefs}

It is impossible to fully understand the complexity of individual families or cultures, but it is important to realize some cultural generalities. Familiaismo, the valuing of family considerations over others including the community, is a strong, almost universal value in the Hispanic community. ${ }^{4}$ The Hispanic patient that is seeking medical care might have already sought help from family resources. ${ }^{5}$ Family involvement in health care is common in the Hispanic population and health care providers are strongly encouraged to support family involvement in medical decisions. This act will reinforce health care provider's support for their patient's belief system. The greatest power in the family is held by the father or oldest male relative. They make health decisions for others in the family. Men are expected to provide for and be in charge of their families. Health providers should understand and comply with patient and family gender roles.

Most Hispanics are Roman Catholic. ${ }^{4}$ Churches are a central aspect of the Hispanic patient's life and community. Religious considerations can be important resources in planning and delivering services. Along with Catholicism is a concurrent beliefs in and use of magico-religious means of dealing with life. ${ }^{5}$ Candles with pictures of saints are very common in the Hispanic population and are often part of altars in the living room or bedrooms. The saints hold specialized meaning for this Hispanic population with each saint having general and specialized religious meaning. Faith and church remain powerful sources of hope and strength in the Hispanic population, especially when facing an illness. Daily rituals of prayer, devotions, and wearing religious medallions are common. ${ }^{4}$ Some Hispanics have unique traditional health beliefs and practices and these are practiced to

\author{
Adam C Schneider \\ Southern Illinois University Edwardsville, Paris Community \\ Hospital, USA
}

Correspondence: Adam C Schneider, Southern Illinois University Edwardsville, Paris Community Hospital, I3980 East I I 30th Road Marshall, IL 6244 I, USA, Tel 2178270349, Email adschne@gmail.com

Received: November 08, 2017 | Published: November 10, 2017

varying degrees. ${ }^{5}$ For example, some Hispanics have belief in hot and cold practices of balancing sickness. Aspects of hot states include hypertension, pregnancy, acid reflux, and diabetes. ${ }^{4}$ Cold states include menstrual craps, colic, and pneumonia. Some Hispanic people will to treat hot states with cold remedies and the opposite for cold states. There are numerous significant interactions with herbal remedies and drugs seen in anesthesia including Echinacea, ephedra, garlic, ginko, kava, and St. John's wort. ${ }^{6}$ Having an understanding of these is helpful in assessing and understanding Hispanic patients and communities. Many persons who follow these practices are reluctant to share their beliefs with nurses or physicians, hence building trust and resisting judgment is essential to practice in these communities..$^{5}$ Disease prevention and health promotion need to encouraged and promoted in Hispanic communities. ${ }^{4}$ With the reluctance to share their beliefs with nurses or physicians, overall limited access to care, and lack of insurance for some Hispanics can lead the Hispanic population to have exaggerated disease states. They could be more acutely ill or have less controlled comorbidities. ${ }^{7}$

Language differences can be a barrier for any healthcare provider. ${ }^{4}$ Most Hispanic people's primary language is Spanish. Many Hispanics are bilingual as well so it is important for the healthcare provider to establish the patient's preferred language. Most hospitals have interpretive services available to hospital employees to ensure informed decision making. It is recommended in a journal article by Hicks ${ }^{4}$ that an interpreter of the same sex as the patient is usually best for the Hispanic population.

\section{Anesthetic considerations}

In the U.S., 18 states contributed to The U.S. Standard Certificate of Live Birth in 2006. ${ }^{8}$ From this data, a total of 60 percent of women in the reporting areas received epidural or spinal anesthesia for pain relief during labor and delivery. Less than half, 47 percent, of Hispanic women received epidural or spinal anesthesia during labor and delivery compared with 69 and 64 percent of non-Hispanic white and African Americans respectively. This could be an indication of the pain tolerance and determination of the Hispanic population. One study, although, brings to light the Hispanic populations resistance to report pain as an reason for underutilizing pain relief measures. ${ }^{9}$ An anesthesia provider should be aware of the possible underreporting of pain and treat vital signs as well as have good communication with their patients. 
The darker pigmentation of the Hispanic population can also have impact on pulse oximetry readings. ${ }^{10} \mathrm{~A}$ study by Feiner et al. ${ }^{10}$ found that pulse oximeters generally overestimate blood oxygenation saturation in patients with hypoxia defined as saturations less than 80 percent. The difference was greatest in dark skinned patients, intermediate in intermediate skinned patients and minimal with light skinned patients. Hispanics were considered intermediate skinned patients.

\section{Conclusion}

Anesthesia providers are no different than other nursing professions when it comes to the importance of cultural competence. The most important aspect of Hispanic culture on anesthesia appears to be pre and postoperative communication and interactions with the patient. Establishing trust by respecting the patient is essential. An anesthetist should be aware of any physical differences and the Hispanic population's belief system which includes Catholicism, familism, Spanish speaking language, and natural remedies. Anesthesia needs to be prepared to use interpretive services to make sure effective patient/ provider communication is present. Sound awareness of differences and respect to beliefs can have a positive impact on the quality of anesthesia delivered. The intraoperative differences of the Hispanic population appear to be limited to possible analgesic needs and pulse oximetry interpretation.

\section{Acknowledgments}

None.

\section{Funding details}

None.

\section{Conflicts of interest}

Authors declare that there is no conflict of interest.

\section{References}

1. US Census Bureau. Asians fastest-growing race or ethnic group in 2012 , Census Bureau reports. 2012.

2. US Census Bureau. State \& county quickfacts:USA. 2013.

3. Young M. Hispanic health information outreach:Recommendations for NLM strategy and tactics. National Library of Medicine. 2001.

4. Hicks D. Cultural competence and the Hispanic population. Medsurg Nurs. 2012;21(5):314-315.

5. Kemp C. Mexican \& Mexican-Americans:Health beliefs \& practices. 2005.

6. Barash PG, Cullen BF, Stoelting RK. Clinical Anesthesia. (6th edn), Lippincott-Williams \& Wilkins, Philadelphia, USA. 2009.

7. Peterson-Iyer K, Culturally competent care for Latino patients: Introduction.

8. Osterman M. Birthstats: Percentage of mothers receiving epidural/spinal anesthesia by age, race, and Hispanic origin of mother: Total of 18 U.S. reporting areas, singletons only, 2006. Birth. 2009;36(4):340-341.

9. Chiauzzi E, Black RA, Frayjo K, et al. Health care provider perceptions of pain treatment in Hispanic patients. Pain Pract. 2011;11(3):267-277.

10. Feiner JR, Severinghaus JW, Bickler PE. Dark skin decreases the accuracy of pulse oximeters at low oxygen saturation:The effects of oximeter probe type and gender. Anesth Analg. 2007;105(6):S19-S23. 